\title{
Avaliação das ações de promoção da saúde desenvolvidas pelos agentes comunitários de saúde ${ }^{\#}$ Performance evaluation of health promotion actions taken by community health workers
}

\author{
Lislaine Aparecida Fracolli* \\ Maria Fernanda Pereira Gomes** \\ Denise Gonçalves Moura Pinheiro*** \\ Danielle Freitas Alvim de Castro****
}

\section{Resumo}

Com base nas análises que sistematicamente foram realizadas sobre o trabalho do Agente Comunitário de Saúde (ACS), o Ministério da Saúde construiu um programa de formação para esse profissional, no qual foram definidas as competências que deveriam compor seu perfil, sendo uma delas a promoção da saúde. O objetivo desta pesquisa foi descrever e representar quantitativamente as ações de promoção da saúde propostas pelo Ministério da Saúde. Trata-se de uma pesquisa quantitativa, que teve como cenário de estudo o Colegiado Gestor Regional (CGR) Alto Capivari, da região de Presidente Prudente-SP. Foram sujeitos desta pesquisa os 97 ACS que trabalhavam nas dezessete equipes de Saúde da Família do CGR. Os dados foram coletados por meio de um formulário tipo Likert, que permitia verificar a ocorrência da realização das ações de promoção da saúde. As ações de promoção da saúde mais realizadas foram as que visam ao desenvolvimento do autocuidado, prevenção de agravos e a identificação de problemas; e as ações menos desenvolvidas foram as de participação social e intersetorialidade, contudo, dentre os desafios para a operacionalização das ações de promoção da saúde mais emancipatórias pelos ACS, verificou-se a atribuição de ações diferentes das propostas pelo Ministério da Saúde; número de famílias além do preconizado e pouca oferta de capacitação para os ACS. O ACS tem potencial e realiza ações importantes de promoção da saúde, contudo essa estratégia deve ser priorizada pelas políticas públicas sociais e de saúde, pois seu desenvolvimento pode impactar positivamente nos indicadores de saúde.

Palavras-chave: Agentes Comunitários de Saúde. Promoção da Saúde. Saúde Pública.

\begin{abstract}
Based on the analyzes that were systematically performed on the work of Community Health Agents (ACS), the Ministry of Health constructed a training program for these professionals in which the skills that should compose their profile were defined, health promotion was one of them. The objective of this research was to describe and quantitatively represent health promotion actions proposed by the Ministry of Health. This is a quantitative research concerning the Collegiate Regional Manager (CGR) Alto Capivari in the Presidente Prudente-SP region. The subjects of this study were 97 ACS working in the 17 teams of Family Health of the CGR. Data were collected through a Likert form that allows verifying the occurrence of health promotion actions. The more frequently performed health promotion actions were the ones aimed at the development of self-care, injury prevention, and problems identification, and the less frequently performed actions were the ones of social participation and intersectoral approach. However, among the operationalization challenges of health promotion actions which were more emancipatory by ACS, the attribution of actions different from those proposed by the Ministry of Health, a number of families beyond the recommended and little offer of training for ACS were verified. ACS have potential and perform important actions to promote health, yet this strategy should be prioritized by social and health public policies since its development can positively impact health indicators.
\end{abstract}

Keywords: Community Health Workers. Health Promotion. Public Health.

\# Pesquisa financiada pela Agência FAPESP; n. do processo: 2010/10193-2.

* Enfermeira. Professora Doutora. Docente do Departamento de Enfermagem em Saúde Coletiva da Escola de Enfermagem da Universidade de São Paulo (EEUSP). São Paulo-SP, Brasil. E-mail: lislaine@usp.br

** Enfermeira. Doutoranda pela Escola de Enfermagem da Universidade de São Paulo (EEUSP). São Paulo-SP, Brasil. E-mail: mferpg@usp.br

*** Fisioterapeuta. Doutoranda pela Escola de Enfermagem da Universidade de São Paulo (EEUSP). São Paulo-SP, Brasil. E-mail: denisep@usp.br

**** Enfermeira. Doutoranda pela Escola de Enfermagem da Universidade de São Paulo (EEUSP). São Paulo-SP, Brasil. E-mail: dani_facastro@hotmail.com

As autoras declaram não haver conflitos de interesse. 


\section{INTRODUC̣ÃO}

A prestação de cuidados primários desenvolvida por membros da comunidade treinados tem sido experimentada em todo o mundo há várias décadas. Esses trabalhadores da saúde recebem diferentes denominações conforme o país onde estão, mas, de uma maneira geral, há uma quantidade de evidências que mostram que a ação desses profissionais pode melhorar a saúde da população. Dentre os países que apresentam tais profissionais, destacamos África Subsaariana (Etiópia, Moçambique e Uganda), Sudeste da Ásia (Bangladesh, Paquistão e Tailândia) e, na América Latina, o Haiti. Nesses locais, as ações mais desenvolvidas por esses profissionais estão voltadas para a saúde materno-infantil, prevenção de HIV / AIDS, prevenção da tuberculose e malária, saúde mental e doenças não transmissíveis ${ }^{1}$.

O desenvolvimento de programas que envolvem trabalhadores comunitários de saúde, com a finalidade de combater os efeitos da pobreza sobre as condições de vida e saúde das comunidades em diversos países, está diretamente relacionado com a Declaração do Milênio das Nações Unidas. Esse documento histórico, criado em 2000 por dirigentes de 189 países, tem como finalidades, entre outras, reduzir para metade o percentual de pessoas que vivem na pobreza extrema, fornecer água potável e educação a todos e inverter a tendência de propagação do HIV / AIDS ${ }^{1}$.

O Brasil, com o intuito de melhorar os indicadores de saúde e as condições de vida da população, utiliza o trabalho de agentes de saúde desde 1987, quando criou o Programa de Agentes Comunitários de Saúde (PACS). Atualmente, o PACS e o Programa Saúde da Família (PSF) dão forma à Estratégia de Saúde da Família, que propõe a reorientação do modelo de atenção à saúde a partir da Atenção Básica ${ }^{2,3}$.

No Brasil, a atividade profissional do ACS foi reconhecida pela Lei n. 10.507, de 10 de junho de 2002, que, entre outras questões, define o exercício profissional do ACS exclusivamente no âmbito do SUS ${ }^{4}$. A partir de 2006, as atividades do ACS passaram a ser regulamentadas pela Lei n. 11.350, que, além de definir suas atribuições, determinou que o ACS tivesse como requisitos para o exercício das suas atividades a conclusão do ensino fundamental e o curso introdutório de formação inicial e continuada ${ }^{5}$.
Com base nas análises que sistematicamente foram realizadas sobre o trabalho do ACS, o Ministério da Saúde construiu um programa de formação para esse profissional, no qual foram definidas as competências que deveriam compor seu perfil. São cinco as competências descritas pelo Ministério da Saúde para o ACS: a) integração da equipe com a população local; b) planejamento e avaliação; c) promoção da saúde; d) prevenção e monitoramento de risco ambiental e sanitário; d) prevenção e monitoramento a grupos específicos e morbidades ${ }^{6}$.

A competência promoção da saúde é formada por um rol de 16 ações que visam à educação em saúde; orientações para o autocuidado; ampliação do conhecimento por parte dos usuários do SUS em relação ao processo saúde-doença; incentivar práticas que promovam a qualidade de vida, a inclusão social e a saúde da população; à promoção de ações intersetoriais e de participação social.

Entre as cinco competências definidas, a Promoção da Saúde parece ser a estratégia que apresenta maior potencial para que o ACS possa ajudar a transformar o modelo de saúde em curso. Tal pressuposto baseia-se no fato de que, ao incorporar o ACS no processo de trabalho, esse profissional possa atuar no fortalecimento e na capacitação da comunidade para o enfrentamento de seus problemas de saúde ${ }^{7}$.

A partir dessas e de outras reflexões realizadas sobre o ACS no Brasil, pode-se dizer que há muito a se pesquisar e a se investir para o aprimoramento desse "novo" profissional de saúde para o SUS.

Recente revisão de literatura sobre o trabalho do ACS na Estratégia Saúde da Família (ESF) e PACS mostrou que esse profissional realiza as ações de saúde que a equipe de saúde delega a ele e que essas ações não são únicas e variam segundo os problemas e necessidades de saúde que as equipes querem superar. Mostrou, ainda, que a prática do ACS está pautada em ser o elo entre a equipe e a comunidade. A prática do ACS é ainda descrita nessas pesquisas como assistencialista, higienista e parece tentar enquadrar as comunidades às normas higienodietéticas relativas ao "viver bem", ao invés de discutir com as comunidades a etiologia dos determinantes de seu processo saúde-doença ${ }^{7,8}$.

Os objetivos desta pesquisa foram descrever e representar quantitativamente as ações de saúde que compõem a competência Promoção da Saúde, desenvolvidas pelos ACS que trabalham nas equipes de saúde da família do CGR Alto Capivari. 


\section{MÉTODO}

Trata-se de um estudo transversal, descritivo e quantitativo. O cenário de estudo escolhido para esta pesquisa foi o Colegiado de Gestão Regional (CGR) de Alto Capivari, pertencente à Rede Regional de Atenção a Saúde (RRAS) 11 de Presidente Prudente. Esse CGR é composto pelos municípios de lepê (7.628 mil habitantes), João Ramalho (4.180 mil habitantes), Nantes (2.750 mil habitantes), Quatá (12.909 mil habitantes) e Rancharia (28.809 mil habitantes). O Colegiado Gestor Regional é um espaço permanente de pactuação, cogestão solidária e cooperação das regiões de saúde que têm como objetivo fundamental garantir o cumprimento dos princípios do Sistema Único de Saúde (SUS). Os Departamentos Regionais de Saúde (DRS) são responsáveis por coordenar as atividades da Secretaria de Estado da Saúde no âmbito regional e promover a articulação intersetorial, com os municípios e organismos da sociedade civil ${ }^{9}$.

Foram sujeitos da pesquisa todos os ACS que trabalhavam nas dezessete equipes de Saúde da Família do CGR de Alto Capivari. Dessa forma, dos 97 ACS que integravam a Saúde da Família do Colegiado em janeiro de 2011, 81 participaram da pesquisa. A coleta de dados deste estudo foi realizada por meio da aplicação de um formulário do tipo Likert aplicado em janeiro de 20117,10,11.

A base desse formulário foram as ações que compõem as cinco competências do ACS, sendo elas: integração da equipe com a população; planejamento e avaliação; promoção da saúde; prevenção e monitoramento de risco ambiental e sanitário; e prevenção e monitoramento a grupos específicos e morbidades. O formulário tipo likert aplicado permite cinco opções de respostas (todos os dias, todas as semanas, todos os meses, raramente e nunca) referentes às ações realizadas pelos ACS.

Os formulários foram digitados em banco de dados eletrônico com o auxílio do programa SPSS 16. Os dados organizados foram analisados segundo a frequência simples das ocorrências e o agrupamento das possibilidades. Com a finalidade de facilitar a análise comparativa dos dados encontrados nesta pesquisa, consideramos as frequências de ocorrências "diariamente" e "raramente".

A presente pesquisa foi aprovada pelo Comitê de Ética em Pesquisa da Escola de Enfermagem da Universidade de São Paulo sob o parecer n. 963/2010 e pelas Secretarias Municipais de Saúde dos cinco municípios.

\section{RESULTADOS E DISCUSSÃO}

A Tabela 1 demonstra que os resultados obtidos acerca da frequência de realização das ações que compõem a competência promoção da saúde, com relação à ação $C 1$ - Identificar a relação entre problemas de saúde e condições de vida, são realizados diariamente em $71,60 \%$ dos casos e raramente em $6,17 \%$ dos casos.

Tabela 1. Frequência das ações de promoção da saúde desenvolvidas no CGR de Alto Capivari. 2011

\begin{tabular}{|c|c|c|c|c|c|}
\hline $\begin{array}{c}\text { Ações de Promoção } \\
\text { da saúde }\end{array}$ & $\begin{array}{c}\text { Todos os } \\
\text { dias }\end{array}$ & Todas as semanas & Todos os meses & Raramente & Nunca \\
\hline C1 & $71,6 \%$ & $12,3 \%$ & $9,9 \%$ & $6,2 \%$ & $0 \%$ \\
\hline $\mathrm{C} 2$ & $56,8 \%$ & $13,6 \%$ & $12,3 \%$ & $17,3 \%$ & $0 \%$ \\
\hline C3 & $3,7 \%$ & $18,5 \%$ & $45,7 \%$ & $29,6 \%$ & $2,5 \%$ \\
\hline C4 & $14,8 \%$ & $25,9 \%$ & $13,6 \%$ & $34,6 \%$ & $11,1 \%$ \\
\hline C5 & $32,1 \%$ & $1,2 \%$ & $11,1 \%$ & $16,0 \%$ & $39,5 \%$ \\
\hline $\mathrm{C} 6$ & $33,3 \%$ & $6,2 \%$ & $23,5 \%$ & $33,3 \%$ & $2,5 \%$ \\
\hline $\mathrm{C} 7$ & $71,6 \%$ & $6,2 \%$ & $14,8 \%$ & $6,2 \%$ & $0 \%$ \\
\hline $\mathrm{C} 8$ & $86,4 \%$ & $3,7 \%$ & $9,9 \%$ & $0 \%$ & $0 \%$ \\
\hline C9 & $86,4 \%$ & $1,2 \%$ & $12,3 \%$ & $0 \%$ & $0 \%$ \\
\hline C10 & $75,3 \%$ & $4,9 \%$ & $13,6 \%$ & $4,9 \%$ & $1,2 \%$ \\
\hline C11 & $25,9 \%$ & $9,9 \%$ & $8,6 \%$ & $40,7 \%$ & $14,8 \%$ \\
\hline C12 & $17,3 \%$ & $4,9 \%$ & $9,9 \%$ & $49,4 \%$ & $18,5 \%$ \\
\hline C13 & $13,6 \%$ & $4,9 \%$ & $23,5 \%$ & $45,7 \%$ & $11,1 \%$ \\
\hline C14 & $49,4 \%$ & $6,2 \%$ & $21,0 \%$ & $22,2 \%$ & $1,2 \%$ \\
\hline C15 & $44,4 \%$ & $2,5 \%$ & $12,3 \%$ & $29,6 \%$ & $11,1 \%$ \\
\hline C16 & $2,5 \%$ & $2,5 \%$ & $16,0 \%$ & $55,6 \%$ & $22,5 \%$ \\
\hline
\end{tabular}

Fonte: elaborada pelos autores a partir dos dados coletados. 
Na pesquisa realizada em São Bernardo do Campo, identificou-se o percentual de realização Para C1 de 58\% diariamente e 11,20\% raramen$\mathrm{te}^{7}$. No município de Marília, encontrou-se o percentual de realização igual a 60,48\% diariamente e $7,18 \%$ raramente ${ }^{11}$. Na pesquisa realizada no município de São Paulo, obteve-se um percentual de realização dessa ação de 43,7\% diariamente e $0 \%$ raramente, e a soma das frequências positivas resultou em um total de $100 \%{ }^{10}$.

A ação C1 (identificar a relação entre problemas de saúde e condições de vida) teve alto percentual de realização no CGR Alto Capivari. Isso indica que os ACS, na visita domiciliar, buscam identificar os problemas e relacioná-los com as condições de vida das famílias e da comunidade que está sob seus cuidados. Esse fato pode ser comprovado pelo percentual de $71,60 \%$ de ACS que referem realizar essa atividade diariamente.

A ação C2 - Identificar exemplos positivos que promovem a saúde na comunidade, segundo os ACS que atuam no CGR Alto Capivari, é realizada num percentual de 56,79\% diariamente e $17,28 \%$ raramente. Em São Bernardo do Campo, o percentual de realização dessa ação nas frequências diariamente e raramente foi, respectivamente, de $51,87 \%$ e $9,96 \%{ }^{7}$. No município de Marília, essas frequências foram, respectivamente, de $57,48 \%$ e $8,38 \%{ }^{11}$. Na pesquisa realizada em São Paulo, identificou-se um percentual de realização para essas frequências de, respectivamente, $25 \%$ e $6,25 \%{ }^{10}$.

A ação C2 (identificar exemplos positivos que promovem a saúde na comunidade) teve alto percentual de realização no CGR Alto Capivari, bem como nos municípios de São Bernardo do Campo, São Paulo e Marília 7,11,10. O desenvolvimento dessa ação refere-se à percepção que os ACS têm dado ao praticarem as visitas domiciliares. Os resultados demonstram que os ACS têm focado o lado positivo dos processos e inter-relações que ocorrem na comunidade. No entanto, de acordo com a pesquisa realizada no município de Sorocoba ${ }^{12}$, os ACS e os demais profissionais que atuam na Saúde da Família, muitas vezes, atuam na comunidade com ações que focam a doença, esquecendo-se de identificar os indícios de saúde e de ações positivas que a comunidade pode estar desenvolvendo ${ }^{7,10,11}$.
Na C3 - Realizar atividades educativas para a comunidade juntamente com o enfermeiro, de acordo com as respostas dos ACS do CGR Alto Capivari, é realizada num percentual de 3,70\% diariamente e 29,62\% raramente. Na pesquisa realizada em São Bernardo do Campo, verificou-se que a frequência de realização dessa ação diariamente foi de $6,22 \%$ e raramente de $30,70 \%{ }^{7}$. Na pesquisa realizada em Marília, obteve se uma frequência de realização dessa ação de 12,57\% diariamente e 26,35\% raramente ${ }^{11}$. Em São Paulo, verificou-se que a frequência de realização dessa ação foi de $0 \%$ diariamente e $50 \%$ raramente ${ }^{10}$.

Em relação à ação $C 4$ - Realizar atividades educativas com outros ACS, os ACS do CGR Alto Capivari afirmaram realizá-la num percentual de $14,81 \%$ diariamente e $34,57 \%$ raramente. No município de São Bernardo do Campo ${ }^{7}$, identificou-se que a frequência de realização dessa ação foi de 4,56\% diariamente e 43,57\% raramente. Em Marília' ${ }^{11}$, obteve-se uma frequência de realização dessa ação de 23,35\% diariamente e $31,14 \%$ raramente. No município de São Paulo ${ }^{10}$, identificou-se que a frequência de realização dessa ação foi de 12,5\% diariamente e 18,75\% raramente.

A ação C5 - Realizar sozinho atividades educativas para a comunidade é realizada no percentual de $32 \%$ diariamente e $16 \%$ raramente pelos ACS que trabalham no CGR Alto Capivari. Na pesquisa realizada em São Bernardo do Campo, encontrou-se uma frequência de realização dessa ação em um percentual de 18,67\% diariamente e $34 \%$ raramente ${ }^{7}$. Em Marília, identificou-se que a frequência de realização dessa ação foi de $18,56 \%$ diariamente e $32,93 \%$ raramente ${ }^{11}$. Na pesquisa realizada no município de São Paulo, identificou-se que a realização dessa ação ocorre em uma frequência de $6,25 \%$ diariamente e $12,5 \%$ raramente $^{10}$.

A ação C6 - Utilizar recursos de informação e comunicação adequados a realidade local é realizada pelos ACS do CGR Alto Capivari num percentual de 33,33\% diariamente e 33,33\% raramente. No município de São Bernardo do Campo, foi identificado que a frequência de realização dessa ação foi de 25,31\% diariamente e $28,63 \%$ raramente ${ }^{7}$. Em Marília, observou-se que a frequência de realização dessa ação foi de 
$22,75 \%$ diariamente e $23,35 \%$ raramente ${ }^{11}$. Na pesquisa realizada no município de São Paulo ${ }^{10}$, identificou-se que a frequência de realização dessa ação foi de $0 \%$ diariamente e $43,75 \%$ raramente.

As ações C3 (realizar atividades educativas para a comunidade juntamente com o enfermeiro), C4 (realizar atividades educativas com outros ACS), C5 (realizar sozinho atividades educativas para a comunidade) e C6 (utilizar recursos de informação e comunicação adequados à realidade local) referem-se às ações de educação em saúde. Observa-se que as ações C4 e C3 apresentam baixa frequência de realização diariamente, segundo os ACS que atuam no CGR Alto Capivari. O desenvolvimento de atividades educativas juntamente com o enfermeiro C3 teve maior frequência de realização mensalmente $(45,7 \%)$, e as atividades educativas com outros ACS C4 tiveram maior frequência de realização semanalmente (25,9\%).

A ação C5 (realizar sozinho atividades educativas para a comunidade) teve um percentual de 55,55\% de não realização, na perspectiva do ACS. Essa informação nos mostra que o ACS não compreende sua prática cotidiana como educativa.

Os dados acima nos mostram que não existe uma definição sobre a perspectiva educativa do ACS. Cabe questionar de fato o que se espera do ACS, que ele seja um visitador sanitário ou que ele seja um educador em saúde. Seja a decisão em uma ou em outra direção, é preciso capacitá-lo para isso. A aposta dessa pesquisa é que ele possa ser um educador em saúde e que atividades educativas desenvolvidas por ele possibilitem a reflexão crítica dos sujeitos e famílias e o empowerment da comunidade.

Na C7 - Estimular os indivíduos e a comunidade a refletirem sobre as suas condições de saúde e doença, é realizada pelos ACS que trabalham no CGR Alto Capivari num percentual de $71,60 \%$ diariamente e $6,17 \%$ raramente. $\mathrm{Na}$ pesquisa realizada no município de São Bernardo do Campo, verificou-se que a frequência de realização dessa ação foi de 71,37\% diariamente e $1,66 \%$ raramente ${ }^{7}$. No município de Marília, observou-se que a frequência de realização dessa ação foi de $65,87 \%$ diariamente e 3,59\% rara- mente $^{11}$. No município de São Paulo, identificou-se que a frequência de realização dessa ação foi de $31,25 \%$ diariamente e $0 \%$ raramente $^{10}$.

A ação C7 (estimular os indivíduos e a comunidade a refletirem sobre as suas condições de saúde e doença) teve alto percentual de realização no CGR Alto Capivari, bem como nos municípios de São Bernardo do Campo, São Paulo e Marília7,10,11. A análise da ação C7 ajuda a qualificar a análise da ação C1, uma vez que os ACS não só identificam a relação entre problema de saúde e condição de vida como estimulam as famílias (os indivíduos) a fazerem também essa reflexão. Esse fato fica evidente quando analisamos a frequência de ocorrência da ação C7. Essa perspectiva aponta para a construção de certa consciência coletiva sobre os determinantes de saúde.

As ações C8 (orientar indivíduos quanto ao autocuidado), C9 (orientar a população quanto a medidas de proteção à saúde), C10 (orientar indivíduos e família quanto a medidas de prevenção de acidentes domésticos) e C14 (orientar a família e/ou portador de necessidades especiais quanto às medidas facilitadoras para a sua máxima inclusão social) parecem integrar cotidianamente o trabalho dos ACS no CGR Alto Capivari, bem como nos municípios de São Bernardo do Campo, São Paulo e Marília ${ }^{7,10,11}$.

As ações C8 - Orientar indivíduos quanto ao autocuidado e C9 - Orientar a população quanto a medidas de proteção à saúde são realizadas pelos ACS do CGR Alto Capivari num percentual de $84,41 \%$ diariamente e $0 \%$ raramente. No município de São Bernardo do Campo, identificou-se que a frequência de realização dessas ações foi de $79,25 \%$ diariamente e $0,83 \%$ raramente ${ }^{7}$. No município de Marília, observou-se que a frequência de realização da ação C8 (orientar indivíduos quanto ao autocuidado) foi de $79 \%$ diariamente e $0 \%$ raramente e que a frequência de realização da ação C9 (orientar a população quanto a medidas de proteção à saúde) foi de $83,23 \%$ diariamente e $0,60 \%$ raramente ${ }^{11}$. Na pesquisa realizada em São Paulo, verificou-se que a frequência de realização da ação C8 foi de 37,25\% diariamente e $0 \%$ raramente, e a frequência de realização da ação C9 foi de 50\% diariamente e $0 \%$ raramente ${ }^{10}$. 
A ação C10 - Orientar indivíduos e família quanto a medidas de prevenção de acidentes domésticos é realizada num percentual de $75,3 \%$ diariamente e 4,94\% raramente, de acordo com as respostas dos ACS do CGR Alto Capivari. Na pesquisa realizada no município de São Bernardo do Campo, identificou-se que essa ação é realizada em uma frequência de $62,24 \%$ diariamente e $6,22 \%$ raramente $^{7}$. Na pesquisa realizada no município de Marília, observou-se que a frequência de realização dessa ação foi de $64,67 \%$ diariamente e $8,38 \%$ raramente ${ }^{11}$. Em São Paulo, verificou-se que a frequência de realização dessa ação foi de 43,75\% diariamente e $0 \%$ raramente ${ }^{10}$.

A ação C14 - Orientar a família e/ou portador de necessidades especiais quanto às medidas facilitadoras para a sua máxima inclusão social é realizada pelos ACS que trabalham no CGR Alto Capivari num percentual de 49,38\% diariamente e $22,22 \%$ raramente. Na pesquisa realizada no município de São Bernardo do Campo, verificou-se que a frequência de realização dessa ação foi de $31,95 \%$ diariamente e $26,14 \%$ raramente ${ }^{7}$. No município de Marília, observou-se que a frequência de realização dessa ação foi de 44,31\% diariamente e $22,22 \%$ raramente ${ }^{11}$. Na pesquisa realizada em São Paulo, identificou-se que a frequência de realização dessa ação foi de 18,75\% diariamente e $18,75 \%$ raramente ${ }^{10}$.

As ações C8 (orientar indivíduos quanto ao autocuidado), C9 (orientar a população quanto a medidas de proteção à saúde), C10 (orientar indivíduos e família quanto a medidas de prevenção de acidentes domésticos) e C14 (orientar a família e/ou portador de necessidades especiais quanto às medidas facilitadoras para a sua máxima inclusão social) parecem integrar cotidianamente o trabalho dos ACS no CGR Alto Capivari, bem como nos municípios de São Bernardo do Campo, São Paulo e Marília ${ }^{7,10,11}$.

A ação C11 - Propor ações que utilizem as diversas secretarias existentes no município é realizada num percentual de $25,92 \%$ diariamente e $40,74 \%$ raramente pelos ACS que trabalham no CGR Alto Capivari. No município de São Bernardo do Campo, observa-se que a frequência de realização dessa ação foi de $15,77 \%$ diariamente e 46,89\% raramente ${ }^{7}$. Em Marília, identificou-se que a frequência de realização dessa ação foi de $11,97 \%$ diariamente e $43,71 \%$ raramente ${ }^{11}$. Na pesquisa realizada no município de São Paulo, verificou-se que a frequência de realização dessa ação foi de $6,25 \%$ diariamente e $37,5 \%$ raramente ${ }^{10}$.

$\mathrm{Na}$ ação C12 - Executar ações que atuam em parceria com outras secretarias existentes no município, $17,28 \%$ dos ACS que trabalham no CGR Alto Capivari referiram realizá-la diariamente, e 49,38\% dos ACS, raramente. Na pesquisa realizada no município de São Bernardo do Campo, identificou-se que a frequência de realização dessa ação foi de 7,47\% diariamente e 56,85\% raramente $^{7}$. No município de Marília, observou-se que a frequência de realização dessa ação foi de $12,57 \%$ diariamente e $46,10 \%$ raramente ${ }^{11}$. Na pesquisa realizada em São Paulo, verificou-se que a frequência de realização dessa ação foi de $6,25 \%$ diariamente e $50 \%$ raramente $^{10}$.

A ação C13-Estabelecerparcerias com creches, asilos, escolas, comerciantes, grupos sociais e outros é realizada pelos ACS que trabalham no CGR Alto Capivari num percentual de 13,58\% diariamente e 45,68\% raramente. Em São Bernardo do Campo, observou-se que a frequência de realização dessa ação foi de $46 \%$ diariamente e 48,96\% raramente 7 . No município de Marília, verificou-se que a frequência de realização dessa ação foi de 19,16\% diariamente e $28,14 \%$ raramente ${ }^{11}$. No município de São Paulo, identificou-se que a frequência de realização dessa ação foi de $6,25 \%$ diariamente e $31,25 \%$ raramente ${ }^{10}$.

As ações C11, C12 e C13 tiveram baixa frequência de realização no CGR Alto Capivari. Observa-se também que a frequência diária de realização dessas ações também foi baixa no CGR Alto Capivari, São Bernardo do Campo, São Paulo e Marília $7,10,11$.

A realização das ações C11, C12, C13 é indicadora da existência de um trabalho intersetorial no município, ou, no caso, na região. Os resultados das frequências de realização dessas ações mostram que não apenas no CGR Alto Capivari, mas em todos os municípios onde esse mapeamento de ações foi realizado, as ações intersetoriais são pouco realizadas. Isso não nos mostra uma incompetência do ACS com isso, mas que o formato de organização das políticas e dos setores públicos no Brasil não privilegia a 
existência de práticas intersetoriais. A construção de proposições nessa direção no Brasil está restrita a algumas experiências municipais.

$\mathrm{Na}$ ação C15 - Apoiar ações sociais de alfabetização de crianças, adolescentes, jovens e adultos, 44,44\% dos ACS que trabalham no CGR Alto Capivari referiram realizá-la diariamente, e 29,63\%, raramente. Em São Bernardo do Campo, observou-se que a frequência de realização dessa ação foi de 41,9\% diariamente e 18,67\% raramente ${ }^{7}$. No município de Marília, verificou-se que a frequência de realização dessa ação foi de $37,12 \%$ diariamente e $26,35 \%$ raramente ${ }^{11}$. $\mathrm{Na}$ pesquisa realizada em São Paulo, identificou-se que a frequência de realização dessa ação foi de $25 \%$ diariamente e $0 \%$ raramente $^{10}$.

$\mathrm{Na}$ ação C16 - Participar de reuniões do conselho local de saúde ou de outros conselhos locais, 2,5\% dos ACS que trabalhavam no CGR Alto Capivari referiram realizá-la diariamente, e 55,6\%, raramente. No município de São Bernardo do Campo, identificou-se que a frequência de realização dessa ação foi de 3,32\% diariamente e 31,53\% raramente ${ }^{7}$. No município de Marília, observou-se que a frequência de realização dessa ação foi de 5,55\% diariamente e 46,10\% raramente ${ }^{11}$. Na pesquisa realizada no município de São Paulo, verificou-se que essa ação foi desenvolvida na frequência de $6,25 \%$ diariamente e $31,25 \%$ raramente $^{10}$.

A ação C16 (participar de reuniões do conselho local de saúde ou de outros conselhos locais) apresentou baixo percentual de realização no CGR Alto Capivari: 16,05\% de realização mensal, enquanto que no município de São Paulo observou-se $18,7 \%$ de participação mensalmen$t^{10}$. Esses resultados mostram uma baixa participação dos ACS nos espaços políticos de decisão sobre a política de saúde regional. Os ACS são profissionais que estimulam a participação e controle social, contudo fica difícil imaginar como eles estimulam essa participação sem estarem envolvidos no processo. Sabe-se que os Conselhos Locais de Saúde são instâncias para o planejamento, execução e avaliação das ações, não só no âmbito local, mas no âmbito municipal, estadual e federal.

O desenvolvimento dessas ações está diretamente relacionado à importância e valorização que os gestores de saúde desses municípios dão à promoção da saúde e quanto a gestão de saúde municipal trabalha em conjunto com as equipes de Saúde da Família, estimulam a realização das ações de promoção da saúde.

Os dados acima apontam que o ACS tem potencial e realiza ações importantes de promoção da saúde. Contudo, ela é uma estratégia que precisa ser priorizada pelas políticas públicas sociais e de saúde, pois seu desenvolvimento pode impactar positivamente nos indicadores de saúde e desenvolvimento do poder sociopolítico das famílias, diminuindo as iniquidades existentes ${ }^{13}$.

Tendo em vista as características de inserção do ACS no serviço de saúde e sua identidade sociocultural que permite uma relação de vínculo e de confiança com as famílias, esse profissional pode ser capacitado a viabilizar ações que desenvolvam as capacidades da comunidade ${ }^{14}$.

\section{CONCLUSÃO}

Analisando o desempenho da competência Promoção da Saúde no CGR Alto Capivari, observou-se que os ACS que trabalham no CGR Alto Capivari realizam em maior percentual as ações que promovem o autocuidado, prevenção de agravos à saúde e a identificação de problemas. As ações de promoção da saúde que visam à participação social e à intersetorialidade são as ações menos realizadas no CGR Alto Capivari pelos ACS, bem como nos municípios de São Bernardo do Campo, Marília e São Paulo.

Os resultados desta pesquisa permitem concluir que as ações de promoção da saúde ainda estão centradas em estratégias de educação em saúde que tomam como foco o controle dos efeitos das condições de vida sobre o processo saúde-doença. Fica evidente que as ações de promoção da saúde que tenham como finalidade reflexões críticas sobre os determinantes da saúde e da doença e o controle social ainda são incipientes, embora exista potencial para que se desenvolvam.

Dentre as dificuldades e desafios para a operacionalização de ações de promoção da saúde mais emancipatórias por parte dos ACS, destacam-se a atribuição de ações fora do contexto, objetivos e pressupostos da ESF aos ACS; núme- 
ro de famílias além do preconizado; políticas e gestão de saúde locais que não estimulam ações emancipatórias e de controle social; pouca oferta de capacitação para a promoção da saúde para os profissionais da ESF.

Os ACS apresentam características importantes e particulares, como a identidade cultural com a comunidade onde trabalham, que possibilita a eles ter maior potencial para desenvolver ações que promovam a saúde e fortaleçam a co- munidade para o enfrentamento dos problemas e necessidades de saúde.

Este estudo aponta para a necessidade de pesquisas relativas ao trabalho dos ACS e a operacionalização da competência Promoção da Saúde. Existem ainda muitos pontos obscuros do trabalho do ACS que precisam ser analisados, como questões éticas e políticas que se fazem presentes para o fortalecimento das comunidades brasileiras.

\section{REFERÊNCIAS}

1. WHO. World Health Organization. Global Experience of Community Health Workers for Delivery of Health Related Millennium Development Goals: A Systematic Review, Country Case Studies, and Recommendations for Integration into National Health Systems. Pakistan; 2010.

2. Ávila MMM. O Programa de Agentes Comunitários de Saúde no Ceará: o caso de Uruburetama. Ciên Saúde Colet. 2011;16(1):349-60.

3. Brasil. Ministério da Saúde. Secretaria de Políticas de Saúde. O trabalho do Agente Comunitário de Saúde. Brasília; 2000.

4. Brasil. Lei n. 10.507, de 10 de julho de 2002. Cria a profissão de Agente Comunitário de Saúde e dá outras providências. Diário Oficial da União, Brasília, 11 jun. 2002; Seção 1, p. 1.

5. Brasil. Lei n. 11.350, de 05 de outubro de 2006. Regulamenta o §5o do art. 198 da Constituição, e dispõe sobre o aproveitamento de pessoal amparado pelo parágrafo único do art. $2^{\circ}$ da Emenda Constitucional n. 51 , de 14 de fevereiro de 2006, e dá outras providências. Diário Oficial da União, Brasília, 06 out. 2006; Seção 1, p. 1-2.

6. Brasil. Ministério da Saúde. Ministério da Educação. Referencial curricular para o curso técnico de agente comunitário de saúde: área profissional. Brasília: Ministério da Saúde; 2004.

7. Santos LPGS. A atuação do agente comunitário de saúde em São Bernardo do Campo: possibilidades e limites para a promoção da saúde [dissertação]. São Paulo: Escola de Enfermagem, Universidade de São Paulo; 2005.

8. Lunardelo SR. O trabalho do agente comunitário de saúde nos núcleos de saúde da família em Ribeirão Preto-SP [dissertação]. Ribeirão Preto (SP): Escola de Enfermagem da Universidade de São Paulo; 2004.

9. Ermel CE, Bigio FM, Evangelista AFR, Gomes MFP, Fracolli LA. Algumas perspectivas para análise da gestão da saúde no estado de São Paulo (Brasil). Ciên Saúde Colet. 2011;16(3):1899-906.

10. Almeida EZ. As concepções do agente comunitário de saúde sobre promoção da saúde [dissertação]. São Paulo: Escola de Enfermagem da Universidade de São Paulo; 2008.

11. Pinto AAM. As potencialidades do agente comunitário de saúde na efetivação da promoção da saúde: uma análise das suas ações no município de Marília-SP [dissertação]. São Paulo: Escola de Enfermagem da Universidade de São Paulo; 2010.

12. Gomes KO, Cotta RMM, Cherchiglia ML, Mitre SM, Batista RS. A práxis do agente comunitário de saúde no contexto do programa saúde da família: reflexões estratégicas. Saúde Soc. 2009;18(4):744-55.

13. Johnson CD, Noyes J, Haines A, Thomas K, Stockport C, Ribas AN, et al. Learning from the Brazilian Community Health Worker Model in North Wales. Globalization Health. 2013;9(25):1-5.

14. Mascarenhas $\mathrm{CHM}$, Prado FO, Fernandes MH. Fatores associados à qualidade de vida de Agentes Comunitários de Saúde. Ciên Saúde Colet. 2013;18(5):1375-86. 